\title{
苯乙烯一锅法合成氨基醇
}

\author{
何淑旺 ${ }^{a}$ 颜世强 ${ }^{b, c}$ 郭 伟 ${ }^{c}$ 翟光喜*,a 张 伟 $*, b$ \\ ( ${ }^{a}$ 山东大学药学院 济南 250012) \\ ${ }^{b}$ 复旦大学药学院 上海 201203) \\ ( ${ }^{c}$ 山东达因海洋生物制药股份有限公司 山东威海 264300)
}

\begin{abstract}
摘要 报道了一种在丙酮和水的混合溶液中 1,3-二澳-5,5-二甲基海因(DBH)催化烯烃两步一锅法合成氨基醇衍生物的 新方法. 室温条件下, 苯乙烯衍生物首先与 $\mathrm{DBH}$ 反应 $0.5 \sim 2.0 \mathrm{~h}$, 然后与胺反应，最终能以 $64 \% \sim 88 \%$ 的收率得到相应 的氨基醇. 采用该方法以 $77 \%$ 的收率完成了 $\beta 2$ 受体激动剂妥洛特罗克级的一锅法制备.

关键词＼cjkstart氨基醇; 一锅法合成; 1,3-二溴-5,5-二甲基海因; 苯乙烯
\end{abstract}

\section{One-Pot Synthesis of Amino Alcohols from Styrenes}

\author{
He, Shuwang ${ }^{a} \quad$ Yan, Shiqiang ${ }^{b, c} \quad{\text { Guo, } \mathrm{Wei}^{c} \quad \text { Zhai, Guangxi }}^{*, a} \quad$ Zhang, Wei ${ }^{*, b}$ \\ $\left({ }^{a}\right.$ College of Pharmacy, Shandong University, Jinan 250012) \\ ( ${ }^{b}$ School of Pharmacy, Fudan University, Shanghai 201203) \\ ( ${ }^{c}$ Shandong Dyne Marine Biopharmaceutical Co., Ltd., Weihai, Shandong 264300)
}

\begin{abstract}
A two-step one-pot procedure for the synthesis of amino alcohols mediated by 1,3-dibromo-5,5-dimethylhydantoin $(\mathrm{DBH})$ in aqueous acetone solution was developed. Styrene derivatives were treated with DBH at room temperature for $0.5 \sim$ $2.0 \mathrm{~h}$ followed by the addition of amine, affording the corresponding amino alcohols in $64 \% \sim 88 \%$ yields. Tulobuterol, a widely used $\beta 2$-adrenergic agonist, was prepared by this protocol in gram scale with the yield of $77 \%$.

Keywords amino alcohol; one-pot synthesis; 1,3-dibromo-5,5-dimethylhydantoin; styrene
\end{abstract}

\section{Introduction}

Amino alcohols are very important motifs frequently found in many natural products ${ }^{[1-6]}$ and synthetic pharmacologically active molecules. ${ }^{[7-10]}$ They are also widely used for the design of chiral ligands and auxiliaries for asymmetric synthesis (Figure 1). ${ }^{[1-12]}$ Given their prominent roles within organic and medicinal chemistry, a wide variety of synthetic routes to these scaffolds have been developed. ${ }^{[13-17]}$ Among them, hydroxyamination of alkenes is efficient and attractive because the starting materials are inexpensive and readily available. Pioneered by Sharpless and co-workers, ${ }^{[18-26]}$ aminohydroxylation of alkenes has been recognized as a facile access to amino alcohols by using catalysts such as osmium, palladium, copper, iron, platinum and so on.

Recently, our group focused on 1,3-dibromo-5,5-dimethyl hydantoin (DBH) mediated one-pot metal-free trans-

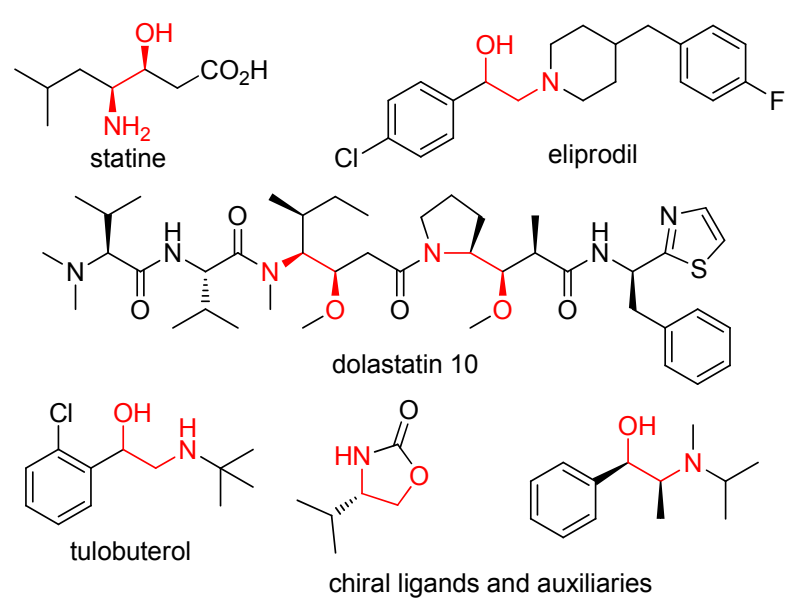

Figure 1 Selected examples bearing amino alcohol scaffold formation of olefins, including the preparations of $\alpha$-bromo/

\footnotetext{
* Corresponding authors. E-mail: professorgxzhai@126.com; zhangw416@fudan.edu.cn Received February 22, 2020; revised May 6, 2020; published online May 27, 2020. Project supported by the National Key Research and Development Program of China (No. 2018YFC0310900) and the National Major Scientific and Technological Special Project for "Significant New Drugs Development” (No. 2018ZX09721003-008-026). 国家重点研究与发展计划(No. 2018YFC0310900)及国家科技重大专项 “重大新药创制” (No. 2018ZX09721003-008-026)资助项目.
} 
amino ketones, ${ }^{[27]} \quad \alpha, \alpha$-dibromoacetophenones, ${ }^{[28]}$ amides, ${ }^{[29]}$ and 2-aminothiazoles. ${ }^{[30]}$ Based on these works, we envisaged that the utility of $\mathrm{DBH}$, a readily available eco-friendly reagent, might be expanded to metal-free synthesis of amino alcohols. Herein, we would like to report a practical one-pot synthesis of amino alcohols from styrenes via treatment with $\mathrm{DBH}$, followed by amination in aqueous medium.

\section{Results and discussion}

As shown in Table 1, our exploration was carried out by using styrene and 4-methylpiperidine as model substrates in different solvents (Entries 1 5). The best result was obtained by using acetone in water (Entry 3), while other solvents such as acetonitrile, dioxane, tetrahydrofuran (THF) and $N, N$-dimethylformamide (DMF), gave inferior results. The yields reduced slightly when increasing or decreasing the amount of diethylene glycol (DBH) (Entries 6 and 7). While using more DBH (Entry 8 vs 3), the yield decreased dramatically due to the over-oxidation of styrene. Much more $\alpha$-bromoketone was detected under this condition, which was produced by excessive DBH. More amines helped to improve the yield, but not significantly (Entry 10 vs 3). On the other hand, 2.0 equiv. of 4-methylpiperidine was insufficient because it could not consume the newly formed bromohydrin intermediates thoroughly (Entry 9). Next, we adjusted the ratio of organic solvent (Entries 11 13) and found that this two-step one-pot transformation worked well in mixture of acetone and water with volume ratio of $2: 1$ (Entry 12).

Having established the optimal reaction conditions, the generality of this protocol with a wide range of styrenes was then explored (Table 2). Both substituted styrenes bearing electron-donating $(\mathbf{2 b} \sim \mathbf{2 d})$ and electron-withdrawing $(\mathbf{2 e} \sim \mathbf{2 j})$ groups worked well with satisfactory yields
(64\% 88\%). Ortho-, meta-, and para-chlorostyrene gave similar results $(\mathbf{2} \mathbf{f} \sim \mathbf{2 h})$. Other amine such as diethyl amine also worked well with this protocol $(\mathbf{2} \mathbf{k} \sim \mathbf{2 n})$. Tulobuterol, a $\beta 2$-adrenergic agonist widely used in the treatment of bronchial asthma and chronic obstructive pulmonary disease, was prepared by using this method in gram scale in good yield (20).

As discussed previously, ${ }^{[27,29,31]}$ a possible mechanism of this two-step one-pot reaction is shown in Scheme 1. The role of water was essential, which was used as reaction media and reactant. ${ }^{[32]}$ Briefly, bromohydrin (3) was generated from styrene with DBH in water, which underwent nucleophilic attack by amine in situ affording desired amino alcohol (2a). Less DBH was inefficient to produce enough bromohydrin intermediate, while excessive DBH oxidized the bromohydrin to undesired $\alpha$-bromo ketone (4), decreasing the total yield of amino alcohol.

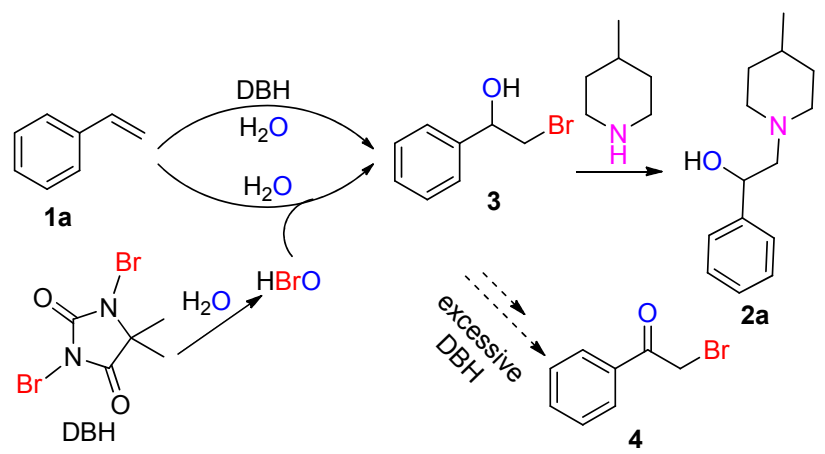

Scheme 1 Possible mechanism

\section{Conclusions}

In conclusion, a convenient procedure for the synthesis of amino alcohols from styrenes mediated by $\mathrm{DBH}$ in aqueous medium has been developed. This metal-free one-pot

Table 1 Optimization of the reaction conditions ${ }^{a}$

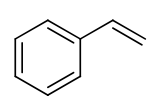

(1) $\mathrm{DBH}$, solvent, r.t., $30 \mathrm{~min}$

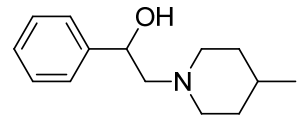

\begin{tabular}{ccccc}
\hline Entry & DBH/equiv. & 4-Methylpiperidine/equiv. & Solvent $(V: V)$ & Isolated yield/\% \\
\hline 1 & 0.55 & 3.0 & Acetonitrile $/ \mathrm{H}_{2} \mathrm{O}(5: 1)$ & 75 \\
2 & 0.55 & 3.0 & Dioxane $/ \mathrm{H}_{2} \mathrm{O}(5: 1)$ & 71 \\
3 & 0.55 & 3.0 & Acetone $/ \mathrm{H}_{2} \mathrm{O}(5: 1)$ & 81 \\
4 & 0.55 & 3.0 & $\mathrm{THF} / \mathrm{H}_{2} \mathrm{O}(5: 1)$ & 43 \\
5 & 0.55 & $\mathrm{DMF} / \mathrm{H}_{2} \mathrm{O}(5: 1)$ & 38 \\
6 & 0.50 & Acetone $/ \mathrm{H}_{2} \mathrm{O}(5: 1)$ & 77 \\
7 & 0.60 & Acetone $/ \mathrm{H}_{2} \mathrm{O}(5: 1)$ & 76 \\
8 & 0.70 & Acetone $/ \mathrm{H}_{2} \mathrm{O}(5: 1)$ & 64 \\
9 & 0.55 & Acetone $/ \mathrm{H}_{2} \mathrm{O}(5: 1)$ & 66 \\
10 & 0.55 & 3.0 & Acetone $/ \mathrm{H}_{2} \mathrm{O}(5: 1)$ & 83 \\
11 & 0.55 & 2.0 & Acetone $/ \mathrm{H}_{2} \mathrm{O}(1: 1)$ & 68 \\
12 & 0.55 & 4.0 & Acetone $/ \mathrm{H}_{2} \mathrm{O}(2: 1)$ & 84 \\
13 & 0.55 & 3.0 & Acetone $/ \mathrm{H}_{2} \mathrm{O}(10: 1)$ & 75 \\
\hline
\end{tabular}

${ }^{a}$ Olefin $(5.0 \mathrm{mmol})$, DBH $(2.75 \mathrm{mmol})$, water $(10 \mathrm{~mL})$ and acetone $(20 \mathrm{~mL})$. 
Table 2 Transformation of terminal alkenes to amino alcohols ${ }^{a}$

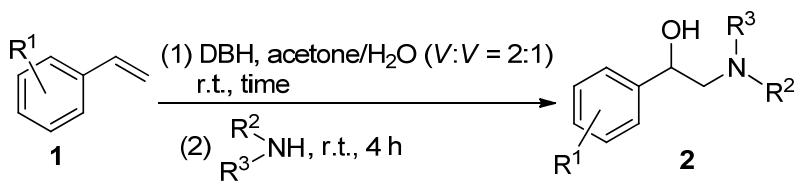

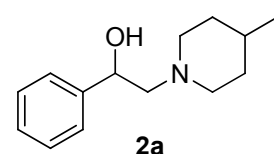

0.5 h $84 \%$

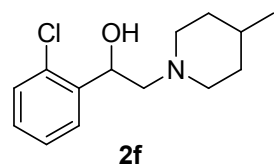

$2.0 \mathrm{~h} \quad 84 \%$<smiles>CCN(CC)CC(O)c1ccccc1</smiles>

0.5 h $\quad 78 \%$

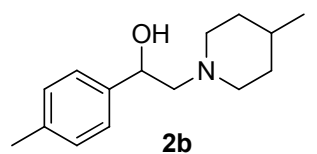

1.0 h $75 \%$

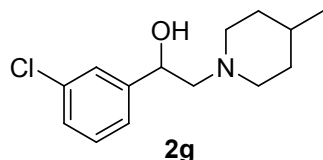

$2.0 \mathrm{~h} \quad 78 \%$<smiles>CCN(CC)CC(O)c1ccc(Cl)cc1</smiles>

2.0 h $\quad 75 \%$<smiles>Cc1cccc(C(O)CN2CCC(C)CC2)c1</smiles>

1.0 h $64 \%$<smiles>CC1CCN(CC(O)c2ccc(Cl)cc2)CC1</smiles>

1.0 h $83 \%$<smiles>CCN(CC)CC(O)c1cccc(Br)c1</smiles>

2.0 h $\quad 68 \%$

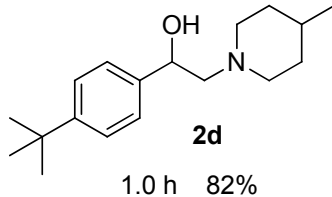<smiles>CC1CCN(CC(O)c2ccc(F)cc2)CC1</smiles>

$1.0 \mathrm{~h} \quad 88 \%$<smiles>CC1CCN(CC(O)c2cccc(Br)c2)CC1</smiles>

$2.0 \mathrm{~h} \quad 85 \%$<smiles>CCN(CC)CC(O)c1cccc([N+](=O)[O-])c1</smiles>

2.0 h $66 \%$<smiles>CC1CCN(CC(O)c2ccc([N+](=O)[O-])cc2)CC1</smiles>

$2.0 \mathrm{~h} \quad 88 \%$

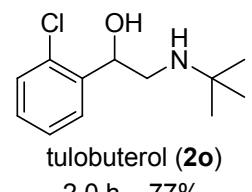

${ }^{a}$ Olefin $(5.0 \mathrm{mmol})$, DBH $(2.75 \mathrm{mmol})$, amine $(15 \mathrm{mmol})$, water $(10 \mathrm{~mL})$ and acetone $(20 \mathrm{~mL})$.

protocol is easy to handle, and facile accessible to various amino alcohols. Bioactivity studies of these newly prepared compounds are currently ongoing in our laboratory and will be reported in due course.

\section{Experimental section}

\subsection{General information}

Nuclear magnetic resonance (NMR) spectra were recorded on a Bruker $500 \mathrm{MHz}$ spectrometer as indicated in the data list. ${ }^{1} \mathrm{H}$ NMR spectra were reported relative to the signal residual $\left(\mathrm{CDCl}_{3}\right.$ at $\left.\delta 7.26\right)$ or TMS. ${ }^{13} \mathrm{C}$ NMR spectra were reported relative to the center line of the $\mathrm{CDCl}_{3}$ triplet at $\delta 77.16$. The MS data were obtained with a Finnigan Mat TSQ-7000 mass spectrometer (ESI-HRMS). Reactions were magnetically stirred and monitored by thin layer chromatography (TLC) with silica gel plates (60F-254) using UV light. Yields refer to pure compounds.

\subsection{General procedure for the synthesis of amino alcohols $2 \mathrm{a} \sim 20$}

To a mixture of olefin $(5.0 \mathrm{mmol})$ in water $(10 \mathrm{~mL})$ and acetone $(20 \mathrm{~mL}), \mathrm{DBH}(2.75 \mathrm{mmol})$ was added. The resultant mixture was stirred at room temperature. After the olefin was consumed completely, amine $(15 \mathrm{mmol})$ was added. After being stirred for another $4 \mathrm{~h}$ at room temperature, the reaction mixture was extracted with ethyl acetate (20 $\mathrm{mL} \times 3)$, washed with brine, and dried over $\mathrm{Na}_{2} \mathrm{SO}_{4}$ subsequently. After being concentrated in vacuo, the crude product was purified by silica column chromatography using $\mathrm{CH}_{2} \mathrm{Cl}_{2} / \mathrm{MeOH}(V: V=15: 1)$ as eluant to give the pure product. For compound 2o, the experiment was carried out on $20 \mathrm{mmol}$ scale (2.78 g of 1-chloro-2-vinylbenzene was used).

2-(4-Methylpiperidin-1-yl)-1-phenylethan-1-ol

White solid, yield $84 \%$. m.p. 83 $84{ }^{\circ} \mathrm{C} ;{ }^{1} \mathrm{H}$ NMR $(500$ $\left.\mathrm{MHz} \mathrm{CDCl}_{3}\right) \delta: 7.35(\mathrm{dt}, J=15.0,7.5 \mathrm{~Hz}, 4 \mathrm{H}), 7.26(\mathrm{t}, J=$ $7.1 \mathrm{~Hz}, 1 \mathrm{H}), 4.71(\mathrm{dd}, J=10.5,3.6 \mathrm{~Hz}, 1 \mathrm{H}), 4.21(\mathrm{~s}, 1 \mathrm{H})$, $3.12(\mathrm{~d}, J=11.4 \mathrm{~Hz}, 1 \mathrm{H}), 2.76(\mathrm{~d}, J=11.6 \mathrm{~Hz}, 1 \mathrm{H}), 2.54 \sim$ $2.37(\mathrm{~m}, 2 \mathrm{H}), 2.29(\mathrm{td}, J=11.5,2.3 \mathrm{~Hz}, 1 \mathrm{H}), 2.00(\mathrm{td}, J=$ 11.6, $2.3 \mathrm{~Hz}, 1 \mathrm{H}), 1.71 \sim 1.56(\mathrm{~m}, 2 \mathrm{H}), 1.44 \sim 1.17(\mathrm{~m}, 3 \mathrm{H})$, $0.94(\mathrm{~d}, J=6.4 \mathrm{~Hz}, 3 \mathrm{H}) ;{ }^{13} \mathrm{C}$ NMR $\left(125 \mathrm{MHz}, \mathrm{CDCl}_{3}\right) \delta$ : $142.50,128.29,127.36,125.85,68.77,66.56,55.59,52.14$, $34.68,34.31,30.73,21.89$; IR (KBr) v: 3114, 2924, 2853, $2812,1451,1073,911 \mathrm{~cm}^{-1}$; ESI-HRMS calcd for $\mathrm{C}_{14} \mathrm{H}_{22}-$ $\mathrm{ON}[\mathrm{M}+\mathrm{H}]^{+} 220.16959$, found 220.16972 .

2-(4-Methylpiperidin-1-yl)-1-( $p$-tolyl)ethan-1-ol (2b): White solid, yield $75 \%$. m.p. 86 88 ${ }^{\circ} \mathrm{C} ;{ }^{1} \mathrm{H}$ NMR $(500$ $\left.\mathrm{MHz}, \mathrm{CDCl}_{3}\right) \delta: 7.25(\mathrm{~d}, J=4.1 \mathrm{~Hz}, 2 \mathrm{H}), 7.15(\mathrm{~d}, J=7.9$ $\mathrm{Hz}, 2 \mathrm{H}), 4.68$ (dd, $J=10.3,3.8 \mathrm{~Hz}, 1 \mathrm{H}), 4.18$ (s, 1H), 3.11 $(\mathrm{d}, J=11.8 \mathrm{~Hz}, 1 \mathrm{H}), 2.76(\mathrm{~d}, J=11.8 \mathrm{~Hz}, 1 \mathrm{H}), 2.47 \sim 2.38$ (m, 2H), $2.33(\mathrm{~s}, 3 \mathrm{H}), 2.28(\mathrm{td}, J=11.5,2.5 \mathrm{~Hz}, 1 \mathrm{H}), 1.99$ $(\mathrm{td}, J=11.6,2.5 \mathrm{~Hz}, 1 \mathrm{H}), 1.70 \sim 1.58(\mathrm{~m}, 2 \mathrm{H}), 1.45 \sim 1.35$ $(\mathrm{m}, 1 \mathrm{H}), 1.33 \sim 1.19(\mathrm{~m}, 2 \mathrm{H}), 0.94(\mathrm{~d}, J=6.4 \mathrm{~Hz}, 3 \mathrm{H}) ;{ }^{13} \mathrm{C}$ NMR (125 MHz, $\left.\mathrm{CDCl}_{3}\right) \delta: 139.48,136.99,129.01,125.84$, $68.65,66.63,55.63,52.14,34.72,34.35,30.77,21.91$, 21.15; IR (KBr) v: 3121, 2924, 2808, 1511, 1448, 1097 $\mathrm{cm}^{-1}$; ESI-HRMS calcd for $\mathrm{C}_{15} \mathrm{H}_{24} \mathrm{ON}[\mathrm{M}+\mathrm{H}]^{+}$ 234.18524, found 234.18533.

2-(4-Methylpiperidin-1-yl)-1-( $m$-tolyl)ethan-1-ol (2c): White solid, yield 64\%. m.p. 66 $68{ }^{\circ} \mathrm{C} ;{ }^{1} \mathrm{H}$ NMR $(500$ $\left.\mathrm{MHz} \mathrm{CDCl}_{3}\right) \delta: 7.29 \sim 7.12(\mathrm{~m}, 4 \mathrm{H}), 4.74(\mathrm{dd}, J=8.9,4.6$ $\mathrm{Hz}, 1 \mathrm{H}), 3.87$ (s, 1H), 3.17 (d, $J=11.5 \mathrm{~Hz}, 1 \mathrm{H}), 2.85$ (d, 
$J=11.8 \mathrm{~Hz}, 1 \mathrm{H}), 2.54 \sim 2.43(\mathrm{~m}, 2 \mathrm{H}), 2.35 \sim 2.31(\mathrm{t}, J=$ $10.4 \mathrm{~Hz}, 4 \mathrm{H}), 2.12 \sim 2.01(\mathrm{~m}, 1 \mathrm{H}), 1.66(\mathrm{t}, J=13.2 \mathrm{~Hz}, 2 \mathrm{H})$, $1.42 \sim 1.24(\mathrm{~m}, 3 \mathrm{H}), 0.95(\mathrm{~d}, J=6.2 \mathrm{~Hz}, 3 \mathrm{H}) ;{ }^{13} \mathrm{C} \mathrm{NMR}$ $\left(125 \mathrm{MHz}, \mathrm{CDCl}_{3}\right) \delta: 139.24,137.11,129.05,128.23$, 126.52, 125.84, 122.98, 68.66 (d, $J=17.8 \mathrm{~Hz}), 66.44$, $55.56,52.27,34.36,34.03,30.61,21.81,21.14$; IR (KBr) $v$ : 3137, 2952, 2924, 2808, 1716, 1448, $825 \mathrm{~cm}^{-1}$; ESI-HRMS calcd for $\mathrm{C}_{15} \mathrm{H}_{24} \mathrm{ON}[\mathrm{M}+\mathrm{H}]{ }^{+}$234.18524, found 234.18542 .

1-(4-(tert-Butyl)phenyl)-2-(4-methylpiperidin-1-yl)ethan-1-ol (2d): White solid, yield 82\%. m.p. 102 103 ${ }^{\circ} \mathrm{C}$; ${ }^{1} \mathrm{H}$ NMR $\left(500 \mathrm{MHz}, \mathrm{CDCl}_{3}\right) \delta: 7.36(\mathrm{~d}, J=8.3 \mathrm{~Hz}, 2 \mathrm{H})$, $7.30(\mathrm{~d}, J=8.3 \mathrm{~Hz}, 2 \mathrm{H}), 4.69$ (dd, $J=9.8,4.4 \mathrm{~Hz}, 1 \mathrm{H}), 4.17$ $(\mathrm{s}, 1 \mathrm{H}), 3.13(\mathrm{~d}, J=11.5 \mathrm{~Hz}, 1 \mathrm{H}), 2.77(\mathrm{~d}, J=11.6 \mathrm{~Hz}, 1 \mathrm{H})$, $2.51 \sim 2.38(\mathrm{~m}, 2 \mathrm{H}), 2.29(\mathrm{td}, J=11.5,2.4 \mathrm{~Hz}, 1 \mathrm{H}), 1.99(\mathrm{td}$, $J=11.6,2.4 \mathrm{~Hz}, 1 \mathrm{H}), 1.74 \sim 1.59(\mathrm{~m}, 2 \mathrm{H}), 1.49 \sim 1.16(\mathrm{~m}$, $3 \mathrm{H}), 1.31$ (s, 9H), $0.94(\mathrm{~d}, J=6.4 \mathrm{~Hz}, 3 \mathrm{H}) ;{ }^{13} \mathrm{C}$ NMR $(125$ $\left.\mathrm{MHz}, \mathrm{CDCl}_{3}\right) \delta: 150.34,139.39,125.67,125.26,68.61$, $66.50,55.66,52.14,34.75,34.54,34.37,31.42,30.80$, 21.94; IR (KBr) v: 3155, 2929, 2953, 2804, 1444, 1097, 829 $\mathrm{cm}^{-1}$; ESI-HRMS calcd for $\mathrm{C}_{18} \mathrm{H}_{30} \mathrm{ON}[\mathrm{M}+\mathrm{H}]{ }^{+}$ 276.23219, found 276.23260.

1-(4-Fluorophenyl)-2-(4-methylpiperidin-1-yl)ethan-1-ol (2e): White solid, yield 88\%. m.p. 97 $99{ }^{\circ} \mathrm{C}$; ${ }^{1} \mathrm{H}$ NMR $\left(500 \mathrm{MHz}, \mathrm{CDCl}_{3}\right) \delta: 7.33(\mathrm{dd}, J=8.5,5.5 \mathrm{~Hz}, 2 \mathrm{H}), 7.02(\mathrm{t}$, $J=8.7 \mathrm{~Hz}, 2 \mathrm{H}), 4.68(\mathrm{dd}, J=10.6,3.5 \mathrm{~Hz}, 1 \mathrm{H}), 4.24(\mathrm{~s}$, $1 \mathrm{H}), 3.10$ (d, $J=11.6 \mathrm{~Hz}, 1 \mathrm{H}), 2.75$ (d, $J=11.7 \mathrm{~Hz}, 1 \mathrm{H})$, 2.45 (dd, $J=12.5,3.6 \mathrm{~Hz}, 1 \mathrm{H}), 2.41 \sim 2.33(\mathrm{~m}, 1 \mathrm{H}), 2.29$ $(\mathrm{td}, J=11.5,2.4 \mathrm{~Hz}, 1 \mathrm{H}), 2.00(\mathrm{td}, J=11.6,2.4 \mathrm{~Hz}, 1 \mathrm{H})$, $1.69 \sim 1.60(\mathrm{~m}, 2 \mathrm{H}), 1.47 \sim 1.36(\mathrm{~m}, 1 \mathrm{H}), 1.33 \sim 1.19(\mathrm{~m}$, 2H), $0.94(\mathrm{~d}, J=6.5 \mathrm{~Hz}, 3 \mathrm{H}) ;{ }^{13} \mathrm{C} \mathrm{NMR}\left(125 \mathrm{MHz}, \mathrm{CDCl}_{3}\right)$ $\delta: 163.13,161.19,138.20(\mathrm{~d}, J=3.0 \mathrm{~Hz}), 127.47(\mathrm{~d}, J=8.0$ $\mathrm{Hz}), 115.20,115.03,68.22$, 66.57, 55.61, 52.14, 34.68, $34.31,30.72$, 21.88; IR (KBr) v: 3103, 2927, 2816, 1601, $1506,1218,1105,1083,835 \mathrm{~cm}^{-1}$; ESI-HRMS calcd for $\mathrm{C}_{14} \mathrm{H}_{21} \mathrm{ONF}[\mathrm{M}+\mathrm{H}]^{+}$238.16017, found 238.16034.

1-(2-Chlorophenyl)-2-(4-methylpiperidin-1-yl)ethan-1-ol (2f): White solid, yield 84\%. m.p. $111 \sim 113{ }^{\circ} \mathrm{C}$; ${ }^{1} \mathrm{H}$ NMR $\left(500 \mathrm{MHz}, \mathrm{CDCl}_{3}\right) \delta: 7.66(\mathrm{~d}, J=7.5 \mathrm{~Hz}, 1 \mathrm{H}), 7.30 \sim 7.25$ $(\mathrm{m}, 2 \mathrm{H}), 7.24 \sim 7.11(\mathrm{~m}, 1 \mathrm{H}), 5.13(\mathrm{dd}, J=10.3,3.0 \mathrm{~Hz}$, $1 \mathrm{H}), 4.27(\mathrm{~s}, 1 \mathrm{H}), 3.16(\mathrm{~d}, J=11.1 \mathrm{~Hz}, 1 \mathrm{H}), 2.79 \sim 2.64(\mathrm{~m}$, 2H), $2.31 \sim 2.22(\mathrm{~m}, 2 \mathrm{H}), 2.05(\mathrm{td}, J=11.7,2.3 \mathrm{~Hz}, 1 \mathrm{H})$, $1.71 \sim 1.55(\mathrm{~m}, 2 \mathrm{H}), 1.43 \sim 1.36(\mathrm{~m}, 1 \mathrm{H}), 1.33 \sim 1.19(\mathrm{~m}$, 2H), $0.94(\mathrm{~d}, J=6.4 \mathrm{~Hz}, 3 \mathrm{H}) ;{ }^{13} \mathrm{C} \mathrm{NMR}\left(125 \mathrm{MHz}, \mathrm{CDCl}_{3}\right)$ $\delta: 139.99,131.71,129.17,128.27,127.27,127.11,65.94$, $64.27,55.62,52.15,34.72,34.33,30.75,21.92$; IR (KBr) $v$ : 3073, 2952, 2972, 2808, 1464, 1435, 1090, 1031, $760 \mathrm{~cm}^{-1}$; ESI-HRMS calcd for $\mathrm{C}_{14} \mathrm{H}_{21} \mathrm{ONCl}[\mathrm{M}+\mathrm{H}]^{+} 254.13062$, found 254.13124 .

1-(3-Chlorophenyl)-2-(4-methylpiperidin-1-yl)ethan-1-ol (2g): White solid, yield 78\%. m.p. $70 \sim 71{ }^{\circ} \mathrm{C}$; ${ }^{1} \mathrm{H}$ NMR $\left(500 \mathrm{MHz} \mathrm{CDCl}_{3}\right) \delta: 7.39(\mathrm{~s}, 1 \mathrm{H}), 7.28 \sim 7.20(\mathrm{~m}, 3 \mathrm{H})$, $4.67(\mathrm{dd}, J=10.7,3.5 \mathrm{~Hz}, 1 \mathrm{H}), 4.26(\mathrm{~s}, 1 \mathrm{H}), 3.12 \sim 3.04(\mathrm{~m}$, $1 \mathrm{H}), 2.81 \sim 2.69(\mathrm{~m}, 1 \mathrm{H}), 2.48(\mathrm{dd}, J=12.4,3.6 \mathrm{~Hz}, 1 \mathrm{H})$, $2.40 \sim 2.25(\mathrm{~m}, 2 \mathrm{H}), 2.01(\mathrm{td}, J=11.6,2.5 \mathrm{~Hz}, 1 \mathrm{H}), 1.73 \sim$ $1.57(\mathrm{~m}, 2 \mathrm{H}), 1.44 \sim 1.35(\mathrm{~m}, 1 \mathrm{H}), 1.33 \sim 1.19(\mathrm{~m}, 2 \mathrm{H}), 0.94$ $(\mathrm{d}, J=6.5 \mathrm{~Hz}, 3 \mathrm{H}) ;{ }^{13} \mathrm{C} \mathrm{NMR}\left(125 \mathrm{MHz}, \mathrm{CDCl}_{3}\right) \delta: 144.77$,
$134.25,129.55,127.44,125.99,123.96,68.20,66.30$, $55.55,52.11,34.64,34.27,30.68,21.86$; IR (KBr) v: 3108, 2928, 2864, 2808, 1574, 1433,1131, 1112, $972 \mathrm{~cm}^{-1}$; ESI-HRMS calcd for $\mathrm{C}_{14} \mathrm{H}_{21} \mathrm{ONCl}[\mathrm{M}+\mathrm{H}]^{+}$254.13062, found 254.13113.

1-(4-Chlorophenyl)-2-(4-methylpiperidin-1-yl)ethan-1-ol (2h): White solid, yield 83\%. m.p. 90 91 ${ }^{\circ} \mathrm{C} ;{ }^{1} \mathrm{H}$ NMR $\left(500 \mathrm{MHz}, \mathrm{CDCl}_{3}\right) \delta: 7.30(\mathrm{~s}, 4 \mathrm{H}), 4.67$ (dd, $J=10.7,3.5$ $\mathrm{Hz}, 1 \mathrm{H}), 4.25(\mathrm{~s}, 1 \mathrm{H}), 3.09$ (d, $J=11.9 \mathrm{~Hz}, 1 \mathrm{H}), 2.78 \sim 2.69$ $(\mathrm{m}, 1 \mathrm{H}), 2.46(\mathrm{dd}, J=12.4,3.5 \mathrm{~Hz}, 1 \mathrm{H}), 2.39 \sim 2.24(\mathrm{~m}$, 2H), $2.00(\mathrm{td}, J=11.6,2.5 \mathrm{~Hz}, 1 \mathrm{H}), 1.70 \sim 1.58(\mathrm{~m}, 2 \mathrm{H})$, $1.50 \sim 1.35(\mathrm{~m}, 1 \mathrm{H}), 1.43 \sim 1.19(\mathrm{~m}, 2 \mathrm{H}), 0.94(\mathrm{~d}, J=6.5$ $\mathrm{Hz}, 3 \mathrm{H}) ;{ }^{13} \mathrm{C}$ NMR $\left(125 \mathrm{MHz}, \mathrm{CDCl}_{3}\right) \delta: 141.09,132.94$, 128.44, 127.21, 68.17, 66.40, 55.58, 52.13, 34.66, 34.29, 30.69, 21.87; IR (KBr) v: 3116, 2924, 2808, 1489, 1135, $1108,829 \mathrm{~cm}^{-1}$; ESI-HRMS calcd for $\mathrm{C}_{14} \mathrm{H}_{21} \mathrm{ONCl}[\mathrm{M}+$ $\mathrm{H}]^{+}$254.13062, found 254.13132.

1-(3-Bromophenyl)-2-(4-methylpiperidin-1-yl)ethan-1-ol (2i): White solid, yield 85\%. m.p. $84 \sim 85{ }^{\circ} \mathrm{C}$; ${ }^{1} \mathrm{H}$ NMR $\left(500 \mathrm{MHz} \mathrm{CDCl}_{3}\right) \delta: 7.54(\mathrm{~s}, 1 \mathrm{H}), 7.38(\mathrm{~d}, J=7.9 \mathrm{~Hz}, 1 \mathrm{H})$, $7.28(\mathrm{~d}, J=7.7 \mathrm{~Hz}, 1 \mathrm{H}), 7.20(\mathrm{t}, J=7.8 \mathrm{~Hz}, 1 \mathrm{H}), 4.66(\mathrm{dd}$, $J=10.7,3.5 \mathrm{~Hz}, 1 \mathrm{H}), 4.26(\mathrm{~s}, 1 \mathrm{H}), 3.09(\mathrm{~d}, J=11.8 \mathrm{~Hz}$, $1 \mathrm{H}), 2.74(\mathrm{~d}, J=11.7 \mathrm{~Hz}, 1 \mathrm{H}), 2.48$ (dd, $J=12.4,3.6 \mathrm{~Hz}$, $1 \mathrm{H}), 2.39 \sim 2.26(\mathrm{~m}, 2 \mathrm{H}), 2.01(\mathrm{td}, J=11.6,2.4 \mathrm{~Hz}, 1 \mathrm{H})$, $1.71 \sim 1.60(\mathrm{~m}, 2 \mathrm{H}), 1.43 \sim 1.37(\mathrm{~m}, 1 \mathrm{H}), 1.33 \sim 1.19(\mathrm{~m}$, 2H), $0.94(\mathrm{~d}, J=6.5 \mathrm{~Hz}, 3 \mathrm{H}) ;{ }^{13} \mathrm{C} \mathrm{NMR}\left(125 \mathrm{MHz}, \mathrm{CDCl}_{3}\right)$ $\delta: 145.03,130.38,129.87,128.90,124.44,122.50,68.14$, $66.31,55.55,52.10,34.64,34.27,30.68,21.87$; IR (KBr) v: 3108, 2926, 2866, 2808, 1569, 1429, 1131, 1112, $695 \mathrm{~cm}^{-1}$; ESI-HRMS calcd for $\mathrm{C}_{14} \mathrm{H}_{21} \mathrm{ONBr}[\mathrm{M}+\mathrm{H}]^{+}$298.08010, found 298.08154.

2-(4-Methylpiperidin-1-yl)-1-(4-nitrophenyl)ethan-1-ol (2j): White solid, yield 88\%. m.p. 99 101 ${ }^{\circ} \mathrm{C} ;{ }^{1} \mathrm{H}$ NMR $\left(500 \mathrm{MHz}, \mathrm{CDCl}_{3}\right) \delta: 8.19(\mathrm{~d}, J=8.7 \mathrm{~Hz}, 2 \mathrm{H}), 7.55(\mathrm{~d}, J=$ $8.7 \mathrm{~Hz}, 2 \mathrm{H}), 4.80$ (dd, $J=10.6,3.6 \mathrm{~Hz}, 1 \mathrm{H}), 4.42$ (s, 1H), $3.10(\mathrm{~d}, J=11.2 \mathrm{~Hz}, 1 \mathrm{H}), 2.74$ (d, $J=11.2 \mathrm{~Hz}, 1 \mathrm{H}), 2.55$ (dd, $J=12.4,3.7 \mathrm{~Hz}, 1 \mathrm{H}), 2.37 \sim 2.29(\mathrm{~m}, 2 \mathrm{H}), 2.05(\mathrm{td}$, $J=11.6,2.5 \mathrm{~Hz}, 1 \mathrm{H}), 1.70 \sim 1.63(\mathrm{~m}, 2 \mathrm{H}), 1.44 \sim 1.37(\mathrm{~m}$, $1 \mathrm{H}), 1.34 \sim 1.18(\mathrm{~m}, 2 \mathrm{H}), 0.95(\mathrm{~d}, J=6.5 \mathrm{~Hz}, 3 \mathrm{H}) ;{ }^{13} \mathrm{C}$ NMR (125 MHz, $\left.\mathrm{CDCl}_{3}\right) \delta: 150.34,147.23,126.44,123.55$, $68.07,65.93,55.50,52.11,34.59,34.23,30.61,21.81$; IR (KBr) v: 3078, 2946, 2933, 1378, 1340, 1075, $702 \mathrm{~cm}^{-1}$; ESI-HRMS calcd for $\mathrm{C}_{14} \mathrm{H}_{21} \mathrm{O}_{3} \mathrm{~N}_{2}[\mathrm{M}+\mathrm{H}]^{+}$265.15467, found 265.15491.

2-(Diethylamino)-1-phenylethan-1-ol (2k): Colorless viscous liquid, yield $78 \%$. ${ }^{1} \mathrm{H}$ NMR $\left(\mathrm{CDCl}_{3}, 400 \mathrm{MHz}\right) \delta$ : $7.46 \sim 7.23(\mathrm{~m}, 5 \mathrm{H}), 4.64(\mathrm{dd}, J=10.6,3.5 \mathrm{~Hz}, 1 \mathrm{H}), 3.74(\mathrm{~s}$, $1 \mathrm{H}), 2.74(\mathrm{dq}, J=14.5,7.3 \mathrm{~Hz}, 2 \mathrm{H}), 2.66 \sim 2.52(\mathrm{~m}, 3 \mathrm{H})$, 2.45 (dd, $J=12.7,10.7 \mathrm{~Hz}, 1 \mathrm{H}), 1.07$ (t, $J=7.1 \mathrm{~Hz}, 6 \mathrm{H})$; ${ }^{13} \mathrm{C}$ NMR $\left(\mathrm{CDCl}_{3}, 150 \mathrm{MHz}\right) \delta$ : 142.67, 128.31,127.36, 125.84, 69.23, 61.84, 46.90, 11.99; IR (KBr) v: 3105, 2928, 2808, 1504, 1408, $1097 \mathrm{~cm}^{-1}$; ESI-HRMS calcd for $\mathrm{C}_{12} \mathrm{H}_{20} \mathrm{ON}[\mathrm{M}+\mathrm{H}]^{+}$194.15394, found 194.15408.

1-(4-Chlorophenyl)-2-(diethylamino)ethan-1-ol (2l): Colorless viscous liquid, yield 75\%. ${ }^{1} \mathrm{H}$ NMR $\left(\mathrm{CDCl}_{3}, 400\right.$ $\mathrm{MHz}) \delta: 7.33(\mathrm{dd}, J=8.5,5.5 \mathrm{~Hz}, 2 \mathrm{H}), 7.01(\mathrm{t}, J=8.7 \mathrm{~Hz}$, 2H), 4.60 (dd, $J=10.6,3.4 \mathrm{~Hz}, 1 \mathrm{H}), 4.01$ (s, 1H), 2.70 (dt, 
$J=14.4,7.2 \mathrm{~Hz}, 2 \mathrm{H}), 2.62 \sim 2.45(\mathrm{~m}, 3 \mathrm{H}), 2.38(\mathrm{dd}, J=$ $12.8,10.6 \mathrm{~Hz}, 1 \mathrm{H}), 1.06(\mathrm{td}, J=7.2,2.6 \mathrm{~Hz}, 6 \mathrm{H}) ;{ }^{13} \mathrm{C} \mathrm{NMR}$ $\left(\mathrm{CDCl}_{3}, 150 \mathrm{MHz}\right) \delta: 141.26,132.91,128.43,127.18$, 68.62, 61.69, 46.89, 11.97; IR (KBr) v: 3128, 2904, 2808, $1507,1428,1087 \mathrm{~cm}^{-1}$; ESI-HRMS calcd for $\mathrm{C}_{12} \mathrm{H}_{19} \mathrm{ONCl}$ $[\mathrm{M}+\mathrm{H}]^{+}$228.11497, found 228.11511.

1-(3-Bromophenyl)-2-(diethylamino)ethan-1-ol (2m): Colorless viscous liquid, yield 68\%. ${ }^{1} \mathrm{H} \mathrm{NMR}\left(\mathrm{CDCl}_{3}, 400\right.$ MHz) $\delta: 7.53(\mathrm{~s}, 1 \mathrm{H}), 7.38 \sim 7.34(\mathrm{~m}, 1 \mathrm{H}), 7.26(\mathrm{~d}, J=7.7$ $\mathrm{Hz}, 1 \mathrm{H}), 7.17$ (t, $J=7.8 \mathrm{~Hz}, 1 \mathrm{H}), 4.57$ (dd, $J=10.5,3.6 \mathrm{~Hz}$, $1 \mathrm{H}), 4.19(\mathrm{~s}, 1 \mathrm{H}), 2.75 \sim 2.47(\mathrm{~m}, 5 \mathrm{H}), 2.36(\mathrm{dd}, J=12.8$, $10.5 \mathrm{~Hz}, 1 \mathrm{H}), 1.03(\mathrm{t}, J=7.1 \mathrm{~Hz}, 6 \mathrm{H}) ;{ }^{13} \mathrm{C} \mathrm{NMR}\left(\mathrm{CDCl}_{3}\right.$, $150 \mathrm{MHz}) \delta$ : 145.17, 130.22, 129.76, 128.75, 124.33, 122.39, 68.54, 61.54, 46.79, 11.89; IR (KBr) v: 3111, 2914, 2805, 1507, 1438, $1092 \mathrm{~cm}^{-1}$; ESI-HRMS calcd for $\mathrm{C}_{12} \mathrm{H}_{19} \mathrm{ONBr}[\mathrm{M}+\mathrm{H}]^{+}$272.06445, found 272.06450.

2-(Diethylamino)-1-(3-nitrophenyl)ethan-1-ol (2n): Colorless viscous liquid, yield $66 \% .{ }^{1} \mathrm{H}$ NMR $\left(\mathrm{CDCl}_{3}, 400\right.$ MHz) $\delta: 8.26(\mathrm{~s}, 1 \mathrm{H}), 8.15 \sim 8.06(\mathrm{~m}, 1 \mathrm{H}), 7.73(\mathrm{~d}, J=7.7$ $\mathrm{Hz}, 1 \mathrm{H}), 7.52$ (dd, $J=10.5,5.3 \mathrm{~Hz}, 1 \mathrm{H}), 4.74$ (dd, $J=10.4$, $3.7 \mathrm{~Hz}, 1 \mathrm{H}), 4.43(\mathrm{~s}, 1 \mathrm{H}), 2.88 \sim 2.67(\mathrm{~m}, 3 \mathrm{H}), 2.60$ (dq, $J=13.9,7.0 \mathrm{~Hz}, 2 \mathrm{H}), 2.40$ (dd, $J=12.8,10.5 \mathrm{~Hz}, 1 \mathrm{H}), 1.07$ $(\mathrm{t}, J=7.1 \mathrm{~Hz}, 6 \mathrm{H}) ;{ }^{13} \mathrm{C} \mathrm{NMR}\left(\mathrm{CDCl}_{3}, 150 \mathrm{MHz}\right) \delta: 148.15$, $145.07,131.82,129.07,122.07,120.61,68.31,61.28$, 46.73, 11.76; IR (KBr) v: 3101, 2929, 2812, 1499, 1438, $1085 \mathrm{~cm}^{-1}$; ESI-HRMS calcd for $\mathrm{C}_{12} \mathrm{H}_{19} \mathrm{O}_{3} \mathrm{~N}_{2}[\mathrm{M}+\mathrm{H}]^{+}$ 239.13902, found 239.13911.

2-(tert-Butylamino)-1-(2-chlorophenyl)ethan-1-ol (tulobuterol, 2o): White solid, yield 77\%. m.p. $90 \sim 93{ }^{\circ} \mathrm{C}$ (lit. ${ }^{[33]}$ 91 93 $\left.{ }^{\circ} \mathrm{C}\right) ;{ }^{1} \mathrm{H}$ NMR $\left(500 \mathrm{MHz}, \mathrm{CDCl}_{3}\right) \delta: 7.63$ (dd, $J=7.7,1.3 \mathrm{~Hz}, 1 \mathrm{H}), 7.34 \sim 7.25(\mathrm{~m}, 2 \mathrm{H}), 7.19$ (td, $J=$ 7.7, $1.6 \mathrm{~Hz}, 1 \mathrm{H}), 4.99$ (dd, $J=8.6,3.5 \mathrm{~Hz}, 1 \mathrm{H}), 3.02$ (dd, $J=12.1,3.5 \mathrm{~Hz}, 1 \mathrm{H}), 2.47$ (dd, $J=12.0,8.6 \mathrm{~Hz}, 1 \mathrm{H}), 1.09$ $(\mathrm{s}, 9 \mathrm{H}) ;{ }^{13} \mathrm{C} \mathrm{NMR}\left(125 \mathrm{MHz}, \mathrm{CDCl}_{3}\right) \delta: 140.91,131.63$, 129.12, 128.18, 127.46, 126.91, 68.78, 50.42, 48.12, 29.00; IR $(\mathrm{KBr}) v: 3125,2928,2806,1507,1458,1096 \mathrm{~cm}^{-1}$; ESI-HRMS calcd for $\mathrm{C}_{12} \mathrm{H}_{19} \mathrm{ONCl}[\mathrm{M}+\mathrm{H}]^{+}$228.11497, found 228.11557. The data is agreement with literature reported. ${ }^{[33]}$

Supporting Information ${ }^{1} \mathrm{H}$ NMR and ${ }^{13} \mathrm{C}$ NMR spectra of targets compounds $\mathbf{2} \mathbf{a} \sim \mathbf{2 0}$. The Supporting Information is available free of charge via the Internet at http://siocjournal.cn.

\section{References}

[1] Umezawa, H.; Aoyagi, T.; Morishima, H.; Matauzaki, H.; Hamada, M.; Takeuchi, T. J. Antibiot. 1970, 23, 259.

[2] Rinehart, K. L.; Gloer, J. B.; Cook, J. C.; Mizsak, S. A.; Scahill, T. A. J. Am. Chem. Soc. 1981, 103, 1857.

[3] Shimojima, Y.; Shirai, T.; Baba, T.; Hayashi, H. J. Med. Chem. 1985, 28,3 .

[4] Shimojima, Y.; Hayashi, H. J. Med. Chem. 1983, 26, 1370.

[5] He, A.-W. R.; Cory, J. G. Anticancer Res. 1999, 19, 421.

[6] Bergmeier, S. C. Tetrahedron 2000, 56, 2561.

[7] Efange, S. M. N.; Kamath, A. P.; Khare, A. B.; Kung, M.-P.; Mach, R. H.; Parsons, S. M. J. Med. Chem. 1997, 40, 3905.

[8] Ohta, Y.; Shinkai, I. Bioorg. Med. Chem. 1997, 5, 465.

[9] Tok, J. B.-H.; Rando, R. R. J. Am. Chem. Soc. 1998, 120, 8279.

[10] Hallinan, E. A.; Tsymbalov, S.; Finnegan, P. M.; Moore, W. M.; Jerome, G. M.; Currie, M. G.; Pitzele, B. S. J. Med. Chem. 1998, 41, 775.

[11] Ager, D. J.; Prakash, I.; Schaad, D. R. Chem. Rev. 1996, 96, 835.

[12] Studer, A. Synthesis 1996, 793.

[13] Reetz, M. T. Chem. Rev. 1999, 99, 1121.

[14] Scriven, E.; Turnbull, K. Chem. Rev. 1988, 88, 297.

[15] Knapp, S. Chem. Soc. Rev. 1999, $28,6$.

[16] Donohoe, T. J.; Callens, C. K. A.; Flores, A.; Lacy, A. R.; Rathi, A. H. Chem.-Eur. J. 2011, 17, 58.

[17] Reiser, O.; Nilov, D. Adv. Synth. Catal. 2002, 344, 1169.

[18] Yin, G.; Mu, X.; Liu, G. Acc. Chem. Res. 2016, 49, 2413.

[19] Li, G.; Chang, H.-T.; Sharpless, K. B. Angew. Chem., Int. Ed. Engl. 1996, 35, 451.

[20] Alexanian, E. J.; Lee, C.; Sorensen, E. J. J. Am. Chem. Soc. 2005, 127,7690

[21] Li, J.; Grubbs, R. H.; Stoltz, B. M. Org. Lett. 2016, 18, 5449.

[22] Desai, L. V.; Sanford, M. S. Angew. Chem., Int. Ed. 2007, 46, 5737.

[23] Liu, G.; Stahl, S. S. J. Am. Chem. Soc. 2006, 128, 7179.

[24] Michaelis, D. J.; Ischay, M. A.; Yoon, T. P. J. Am. Chem. Soc. 2008, 130,6610 .

[25] Legnani, L.; Morandi, B. Angew. Chem., Int. Ed. 2016, 55, 2248.

[26] Zeng, T.; Liu, Z.; Schmidt, M. A.; Eastgate, M. D.; Engle, K. M. Org. Lett. 2018, 20, 3853.

[27] Xu, S.; Wu, P.; Zhang, W. Org. Biomol. Chem. 2016, 14, 11389.

[28] Wu, P.; Xu, S.; Xu, H.; Hu, H.; Zhang, W. Tetrahedron Lett. 2017, 58,618

[29] Ma, C.; Fan, G.; Wu, P.; Li, Z.; Zhou, Y.; Ding, Q.; Zhang, W. Org. Biomol. Chem. 2017, 15, 9889.

[30] Ma, C.; Miao, Y.; Zhao, M.; Wu, P.; Zhou, J.; Li, Z.; Xie, X.; Zhang, W. Tetrahedron 2018, 74, 3602.

[31] Wu, C.; Xin, X.; Fu, Z.-M.; Xie, L.-Y.; Liu, K.-J.; Wang, Z.; Li, W.; Yuan, Z.-H.; He, W.-M. Green Chem. 2017, 19, 1983.

[32] Cao, Z.; Zhu, Q.; Lin, Y.-W.; He, W.-M. Chin. Chem. Lett. 2019 , $30,2132$.

[33] Nobuta, T.; Xiao, G.; Ghislieri, D.; Gilmore, K.; Seeberger, P. H. Chem. Commun. 2015, 51, 15133. 